\title{
Office of Technology Transfer at the University as a strategy to promote innovation and competitiveness. Case: Hidalgo State, México.
}

\author{
Oficinas de Transferencia Tecnológica en las Universidades como Estrategia \\ para Fomentar la Innovación y la Competitividad. \\ Caso: Estado de Hidalgo, México
}

Elba M. Pedraza Amador', Judith A. Velázquez Castro²

\begin{abstract}
The economic development of any country is supported by innovations, hence the importance of generating research, development and innovation (R\&D) is an imperative today for the economic development of Mexico and its regions, in the Hidalgo State case where universities serve as their main sources of knowledge, requiring just one instance to protect the scientific and technological results and developed processes to facilitate technology transfer. Therefore this paper addresses the importance of technology transfer and the role played by technology transfer offices as facilitators of these processes in support of the universities to promote innovation in the state of Hidalgo.

El desarrollo económico de cualquier país se encuentra sustentado en las innovaciones, de ahí que la importancia de generar Investigación, Desarrollo e Innovación $(1+D+i)$ sea un imperativo hoy en día para el desarrollo económico de México y sus regiones, en el caso del estado de Hidalgo donde sus Universidades fungen como principales generadores de conocimiento; se requiere de una instancia que proteja los resultados científicos y tecnológicos desarrollados así como que facilite los procesos de transferencia de tecnología. Por lo anterior el presente trabajo aborda la importancia de la transferencia tecnológica así como el rol que desempeñan las OTT como facilitadores de estos procesos en apoyo a las Universidades para potenciar la innovación en el estado de Hidalgo.
\end{abstract}

Keywords: office of technology transfer (ott); university; company; innovation; competitiveness; oficinas de transferencia tecnológica (ott); universidad; empresa; innovación; competitividad.

Universidad Autónoma del Estado de Hidalgo, Escuela Superior de Tizayuca. Postal Adress: Km. 2.5 de la Carretera Federal Tizayuca, Pachuca, Hidalgo. México. C.P. 43800 Phone: (+52) 01 77I 7I 72000 - Extensión: 5700, 57 I0. E-mail: 'e_mariana200I@yahoo.com.mx, 2judith_veca@hotmail.com

ISSN: 07 I8-2724. (http://www.jotmi.org)

Journal of Technology Management \& Innovation (c) Universidad Alberto Hurtado, Facultad de Economía y Negocios. 


\section{Introducción}

En la actualidad, las Universidades latinoamericanas y en caso particular las mexicanas están luchando por ganarse un lugar frente a la globalización por lo que una de sus tareas primordiales en el momento actual es la internacionalización. Desde esta orientación la educación superior ha mostrado interés por realizar cooperación en investigación con diversas instituciones en el ámbito internacional (Gacel, 2009), primordialmente a través de sus cuerpos académicos, investigadores y estudiantes fortaleciendo de esta manera su naturaleza generadora de conocimiento para la solución de los problemas del quehacer humano.

Por otra parte, el sector empresarial ha comenzado a buscar formas de aumentar la eficacia y eficiencia de sus procesos de innovación, a través de la búsqueda de nuevas tecnologías e ideas con el fin de crear valor para el cliente, por lo que están recurriendo a esquemas vinculación con Centros de Investigación y Universidades como fuentes de conocimiento que les permitan obtener insumos para la innovación (ADIAT, 20 10:34).

La necesidad de vincular al sector empresarial con las Universidades, Centros de Investigación y dependencias gubernamentales como un medio para fortalecer las relaciones entre actores del sistema de innovación, para potenciar la competitividad de las empresas y orientar la investigación hacia la satisfacción de necesidades concretas, motiva a las Instituciones de Educación Superior (IES) a proteger los resultados derivados de las acciones en materia de investigación y desarrollo tecnológico a través de organismos que faciliten los procesos de gestión de la propiedad intelectual (PI) y la transferencia de tecnología.

Por lo anterior, con visión global y con miras a la internacionalización, las Universidades reconocen una nueva forma de llevar a cabo el proceso de I+D, de naturaleza cooperativa o en colaboración con otros agentes, así como el diseño de nuevos espacios de transferencia y creación de conocimiento a través de estructuras como las Oficinas de Transferencia Tecnológica (OTT) que permitan dirigir el proceso de transferencia del conocimiento tecno-científico hacia la generación de innovación (Bueno, 2007) así como constituir una forma institucionalizada de transportar y canalizar ideas, invenciones e innovaciones de los investigadores y cuerpos académicos a la industria de la región y a la sociedad (Hülsbeck et al., 20I I) esto dado que las innovaciones y su comercialización están basadas en los efectos de las interacciones entre investigadores e industria, acordes con la tendencia mundial de establecer estructuras organizacionales especializadas, diseñadas para servir de puente entre universidades e industria (Krücken et al., 2007).
El enfoque particular en este trabajo se centra en reconocer el papel de las Oficinas de Transferencia Tecnológica en las Universidades como facilitadoras de los procesos de transferencia tecnológica e impulsores de la innovación que fomentan a su vez la integración, licenciamiento y/o comercialización de tecnologías, la generación y lanzamiento de nuevos negocios para el Estado de Hidalgo, México.

\section{Justificación}

En el contexto de este trabajo, la innovación -como proceso social- es vista como parte inherente para el desarrollo económico de cualquier país, un ejemplo de ello es que la innovación se ha extendido entre las empresas de países en desarrollo asociándose a una mayor productividad y a la dependencia de una red de interacciones con otros actores públicos y privados. (Fagerberg et al., 20I0). Hablar entonces de desarrollo económico es hablar de la capacidad de innovar como resultado de un fenómeno colaborativo entre las empresas, el gobierno y las Universidades lo que implica, que el fomento a redes de colaboración sea necesario para que los distintos actores involucrados en este proceso -Universidades, centros de investigación, empresas, gobierno- se desempeñen eficientemente (Freeman, 1987 y 199I; Nelson, 1993; Metcalf, 1995; Cimoli and Dosi, 1995; Cimoli, 2000).

Lo anterior se enmarca en la política mexicana actual, ya que tanto en el Plan Nacional de Desarrollo (PND) 2007 - 2012 como en otros programas en materia de ciencia y tecnología se establece la importancia de crear y fortalecer un vínculo estrecho entre el sector público, la academia y el sector empresarial para plasmar de manera exitosa los nuevos conocimientos en procesos productivos así como facilitar la adquisición y desarrollo de tecnología para mantener la competitividad de las empresas (Programa Especial de Ciencia, Tecnología e Innovación, PECiTI: 20I2; Plan Nacional de Innovación, 20II); esto como antecedente para que en hoy en día, organismos encargados de analizar el desarrollo de la ciencia, la tecnología y la innovación en México desarrollen propuestas para contribuir con el gobierno en turno al diseño del Programa Especial de Ciencia, Tecnología e Innovación 2012-2037, cuyas metas están enfocadas en formular un marco para promover la innovación dirigida al crecimiento económico buscando conectar con efectividad la I+D existente con el sector productivo, vía divulgación, transferencia del conocimiento e incentivos a la formación de redes así como mejorar la vinculación y el desarrollo de capacidades tecnológicas y de otra naturaleza relacionadas con la innovación (FCCyT, 20I3).

Además, el Comité Intersectorial para la Innovación (CII, 20II) y la Ley de Ciencia y Tecnología (2002) han establecido la necesidad de crear y operar Unidades de Vinculación y Transferencia de Conocimiento con el objetivo de 
ejecutar proyectos en materia de desarrollo tecnológico e innovación y promover la vinculación entre los sectores académico, público y privado.

Por tal motivo, las condiciones actuales en México refrendan la necesidad de vincular al sector empresarial con las Universidades y Centros de Investigación para lograr transferir la tecnología creada por éstos últimos y potenciar la competitividad de las empresas. Lo anterior puede conseguirse a través de las OTT, las cuales serán las responsables de difundir las tecnologías, del mismo modo, serán las intermediarias entre las Universidades y las empresas con el fin último de que todos los agentes involucrados en dicha transferencia se vean beneficiados.

En este contexto, Mowery et al. (2004) y Gurmu et al.(20 I0) revelan que las Universidades producen la mayor cantidad de patentes $y$ que reciben la mayor cantidad de ingresos por licencias y patentes; ya que según datos de la Dirección General de Evaluación Institucional de la UNAM (2012), de I99| a 2009, han sido otorgadas 748 patentes a IES siendo las principales la Universidad Nacional Autónoma de México, la Universidad Autónoma Metropolitana y el Instituto Politécnico Nacional, quienes cuentan con áreas para la transferencia de tecnología.

No obstante, México en comparación con otros países observa una reducida producción científica y tecnológica ya que para el año 2012, la Organización Mundial de la Propiedad Intelectual (OMPI, 20I2) en su informe WIPO
IP Facts and Figures muestra que Estados Unidos, China y Japón son los países con mayor número de solicitudes de patentes concedidas y en cuanto a América Latina encontramos a Brasil por encima de México con un crecimiento del $3.4 \%$ contra el $2.1 \%$ respectivamente. Lo que motiva a mejorar los mecanismos de transferencia de tecnología para generar I+D+i.

Por otra parte, de acuerdo con el Diagnóstico en Ciencia, Tecnología e Innovación 2004-20I0 (FCCyT, 2012) el Producto Interno Bruto (PIB) del Estado de Hidalgo -ubicado en la región centro de México- fue de 9,690 millones de dólares en el 2006, en donde la industria manufacturera aporta cerca del $24 \%$ seguido de los servicios comunales, sociales y personales (18.7\%). En cuanto a las unidades económicas se localizan en el Estado 137 mil, de las cuales el $96.6 \%$ son microempresas. Los tres principales sectores donde se concentran son el sector comercio (81\%), sector transporte (15\%) y $3 \%$ de la industria manufacturera, el resto son empresas del sector minería, agropecuario, electricidad y agua (INEGI, 20I2).

De acuerdo con el Plan Estatal de Desarrollo 201I-2016, el estado de Hidalgo busca "impulsar el diseño, aplicación e implementación de una política pública de ciencia, tecnología innovación que fomente y fortalezca la generación y aplicación del conocimiento, y la vinculación entre los sectores en áreas estratégicas" (PED, 20I I) tales como el sector automotriz, agropecuario, maquila y turismo alternativo; sin embargo se observa la necesidad de realizar diagnósticos

\section{Empresas}

Instituciones No Lucrativas

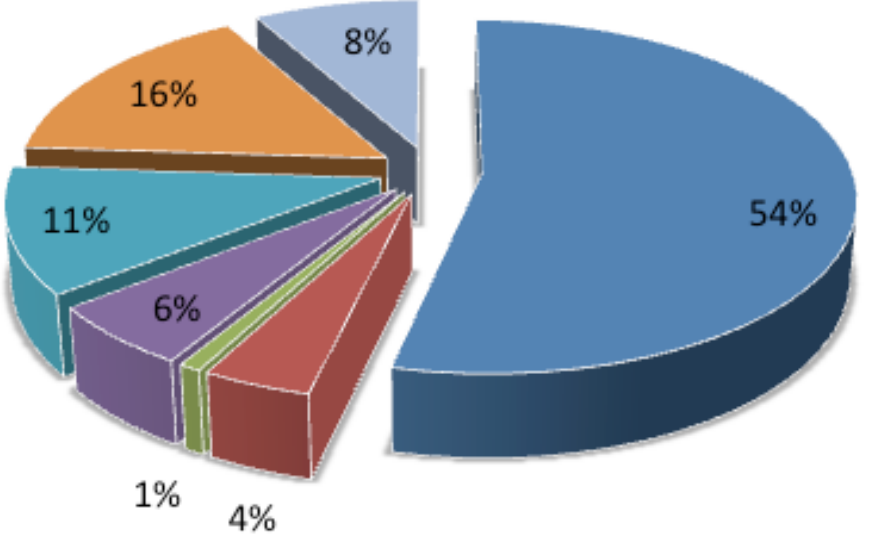

Centros de Investigación (Sede)

- Dependencias de Administración Pública

- Instituciones de Educación Superior (Sede)

- Instituciones de Educación Superior (Subsede)

- Personas físicas con actividad empresarial

Figura I. Integrantes del RENIECYT en Hidalgo Fuente: RENIECYT, 2012.

ISSN: 07 I8-2724. (http://www.jotmi.org) 
de capacidades regionales de innovación con el fin de que el estado pueda asignar recursos de manera armonizada con las fortalezas y áreas de oportunidad de las II regiones que lo conforman aglutinando a 84 municipios.

El índice de Competitividad Estatal ubica al estado de Hidalgo en el número 28 (IMCO, 2008) y el Índice de Innovación Estatal (I2E, 20I0), lo sitúa en el "grupo de baja y escasa innovación" (número 27 a nivel nacional de $3 \mathrm{I}$ entidades). Esto, debido a que la economía de la entidad está basada principalmente en la explotación de sus recursos naturales así como en la utilización de mano de obra barata con actividades de bajo valor agregado, desatendiendo la importancia que reviste la I+D+i para el desarrollo y crecimiento económico, lo que muestra que las bajas capacidades tecnológicas de una región se deben, no sólo a factores parcialmente estáticos como la carencia de infraestructura adecuada $o$ a la falta de instrucción de la mano de obra, sino también al déficit de capital social, asociaciones o redes de empresas que facilitan la coordinación y cooperación para un beneficio mutuo. (Acosta, 1999).

En cuanto a instituciones, centros, organismos y empresas físicas o morales de los diversos sectores que llevan a cabo actividades relacionadas con ciencia, tecnología e innovación, en el Estado existen 121 . Del total, el 27\% son IES, el $54 \%$ son empresas, el $4 \%$ son organismos sin fines de lucro y el $13 \%$ restante son personas físicas con perfil académico, dependencias de la administración pública, personas físicas con actividad empresarial y centros de investigación (ver figura I).
En Hidalgo hay II parques industriales, de los cuales sólo dos son administrados por el estado y el resto son privados, los cuales en su conjunto, aglutinan a 190 empresas distribuidos en 8 municipios. Sin embargo, la infraestructura física existente tiene una fuerte degradación, hay baja disponibilidad de mano de obra, de infraestructura educativa y de vinculación, lo que coloca a la región en desventaja para generar sinergias Universidades-empresas-gobierno repercutiendo a su vez desventajas frente a estados vecinos del centro del país.A pesar de que Hidalgo cuenta con la ventaja de la ubicación geográfica y su conectividad, gracias a la infraestructura carretera reciente que le permite accesibilidad a corredores industriales y de producción de bienes manufacturados del centro-norte del país.

Otro rubro a tomar en consideración para el Estado de Hidalgo en el desempeño del Sistema de Innovación tiene que ver con la infraestructura para la investigación y productividad científica en los que se observa que sólo dos Universidades imparten posgrados pertenecientes al Programa Nacional de Posgrados de Calidad (PNPC) del CONACYT; y sin embargo se nota una escasa productividad en materia de patentes ya que con base en datos del Instituto Mexicano de la Propiedad Industrial se encuentra que se reportó solo una para el año 2008 contrastando con la productividad científica, medida por artículos publicados en revistas indizadas, que reporta 669 artículos de 1998 a 2007 con un promedio de 74 publicaciones cada año (FCCyT, 20I2); lo que denota el bajo nivel de competitividad y potencialidad de innovación del estado.

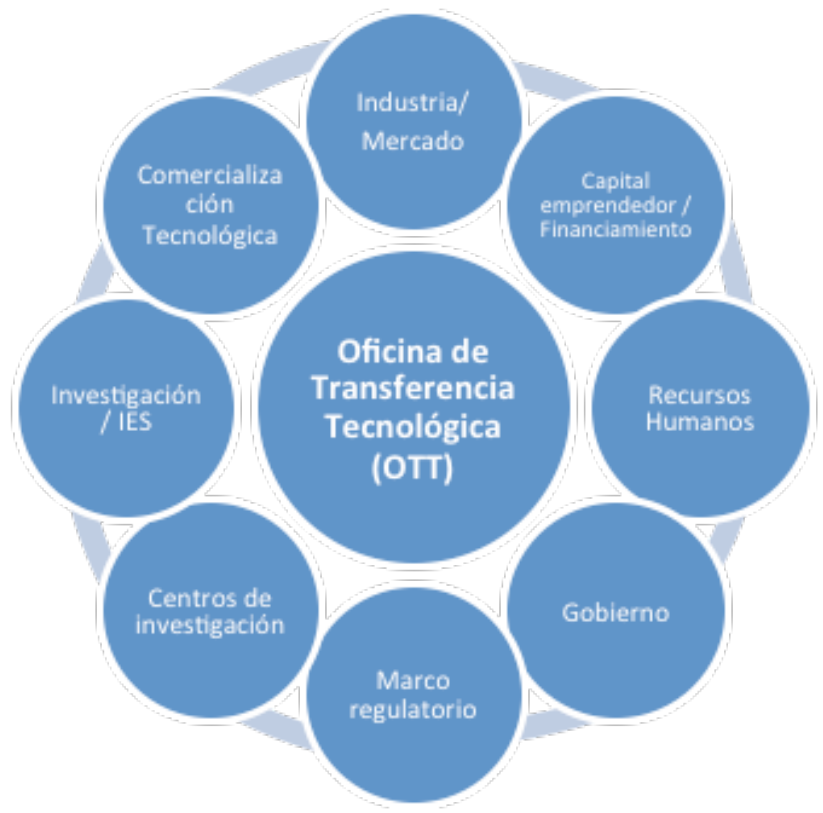

Figura 2.Agentes involucrados en la Transferencia Tecnológica Fuente: CONACYT, 2012.

ISSN: 07 I 8-2724. (http://www.jotmi.org) 


\section{Definición}

La transferencia tecnológica (TT) o de tecnología es definida por la Conferencia de las Naciones Unidas sobre Comercio y Desarrollo (UNCTAD por sus siglas en inglés, 1990) como la "transferencia de conocimiento sistemático, para la elaboración de un producto, la aplicación de un proceso o la prestación de un servicio".

EI CONACYT (2010) define la TT como "el proceso por el cual los resultados de la investigación, desarrollo tecnológico e Innovación, son transferidos a la industria para garantizar su aplicación final en el mercado".

De acuerdo con la Terminología de la Norma Mexicana de Gestión de Tecnología, NMX-GT-00I-IMNC-2007, (IMNC, 2007) la TT es definida como el "proceso mediante el cual se negocia la cesión o licenciamiento de los derechos sobre el capital intelectual".

Para el Consorcio de Laboratorios Federales para la Transferencia de Tecnología de los Estados Unidos (FLC por sus siglas en inglés) la TT es "el proceso de transferencia de habilidades, conocimientos, tecnologías, métodos de fabricación, las muestras de fabricación a los gobiernos o las Universidades y otras instituciones para garantizar que los avances científicos y tecnológicos sean accesibles a una gama más amplia de usuarios que puede seguir desarrollando y explotar esa tecnología en nuevos productos, procesos, aplicaciones, materiales o servicios. La TT está estrechamente relacionada con la transferencia de conocimientos" (Krishen, 20I I:196).

En este moderno concepto pueden identificarse dos actores principales: el proveedor -quien provee la tecnología- y el receptor -quien recibe la tecnología-. Tanto el proveedor como el receptor pueden ser: el Estado, las organizaciones públicas o privadas-, las empresas -nacionales o extranjerasy las Universidades, entre otros (triple hélice). Otro elemento común en estas definiciones, es que se hace hincapié en las actividades de $I+D+i$ de los proveedores y que tienen valor para otros -receptores-. Por lo que la TT pretende compartir y aplicar ese valor generado en donde se necesite. Otro aspecto que no hay olvidar, es que la TT ayuda al desarrollo de recursos humanos en el ámbito de investigación, desarrollo e innovación dado que la creación de capacidades tecnológicas depende de recursos humanos capacitados y del fortalecimiento de la infraestructura necesaria para tal fin. En ese contexto, es indispensable la capacitación, el entretenimiento, la evaluación de las necesidades tecnológicas nacionales y estales, así como la planificación del desarrollo tecnológico para el Estado de Hidalgo para crear una reserva de recursos humanos capacitados y fortalecer la infraestructura tecnológica con la que cuenta actualmente.
Tomando como base las definiciones anteriores, en la figura 2 se distinguen los agentes involucrados en la transferencia tecnológica.

Por ello en el ámbito académico, es de suma importancia entender el proceso de la transferencia de tecnología en la interrelación Universidad-empresa ya que las Universidades no sólo generan nuevo conocimiento a través de la investigación básica sino que también proveen de soporte, conocimiento especializado y servicios a las empresas pues la Universidad no solo esta confinada al proceso de transferencia de conocimiento sino que actúa como un conductor de nuevo conocimiento a través de redes de investigación en los ámbitos nacional e internacional (Hamdan et al., 20l I). Lo anterior permite que el conocimiento desarrollado por las Universidades basado en la investigación científica pueda ser transferido a una o más compañías con múltiples aplicaciones comerciales y de beneficio público, lo que indica la necesidad de mecanismos formales de protección a la propiedad intelectual (Closs, et.al, 2012) con el fin de evitar plagios o de incumplir con lo que establecen las leyes respecto a este ámbito a nivel nacional e internacional.

De igual forma, las Universidades obtienen reconocimiento por la creación de tecnología aplicada, mejora la formación de los estudiantes así como motiva a la creación de programas de posgrado en vinculación con las empresas puesto que se establece vinculación, canales y mecanismos de comunicación además de otorgar incentivos y reconocimientos para el personal académico involucrado (Rodríguez, 2005). Desde el punto de vista de la industria, las razones específicas para la colaboración con la universidad y centros de investigación son la falta de áreas de I+D, acortar el ciclo de vida de los productos y el cambio en la naturaleza de las prioridades de investigación así como establecer mecanismos para el reclutamiento de empleados potenciales.

De ahí que las Oficinas de Transferencia de Tecnología (OTT) se han constituido con el objetivo de promover la interacción de las Universidades con el sector productivo y en especial con las empresas y el gobierno con la finalidad de profesionalizar la gestión, comercialización y transferencia de los resultados de las investigaciones (De Sá Nunes, Agusto y Segatto, 2009) y de la identificación e integración de inversionistas y patrocinadores estratégicos a esas investigaciones; contribuyendo además con la generación de nuevos negocios basados en desarrollos científicos y tecnológicos. Por lo que, una de las condicionantes para generar este círculo virtuoso será la gestión adecuada de la propiedad intelectual (Observatorio virtual de Transferencia Tecnología de España, 20I2; CONACYT, 20I0). 


\section{Objetivo de las OTT}

Las OTT tienen como principal objetivo transferir a la sociedad los conocimientos y tecnologías desarrollados en su entorno de investigación. Suelen formar parte de las Universidades, Centros de Investigación y Parques Científicos y Tecnológicos como las unidades de transferencia de tecnología que ofertan sus soluciones y resultados de investigación.

Por otra parte su misión es ayudar a las Universidades a actuar proactivamente en el proceso de innovación facilitando la conversión de los resultados de investigación de nuevos productos o servicios en beneficio de la sociedad así como de promover el desarrollo económico regional, la creación de empleo y generar recursos financieros complementarios para la misma institución a través de la investigación en colaboración con otros sectores, consultorías y donaciones (Beraza y Rodríguez, 2010).

Sin embargo para que las OTT puedan cumplir con éxito su misión es vital que las Universidades cuenten con profesorado calificado, políticas de promoción, un adecuado sistema de incentivos así como de procesos de gestión de la propiedad intelectual y orientación en la investigación que conformen un entorno adecuado para la transferencia de conocimiento.

La protección de PI debe ser una estrategia que fomenta y agiliza la difusión de nuevos conocimientos en beneficio de Hidalgo. Por lo que, una apropiada política institucional en materia de $\mathrm{Pl}$ es de suma importancia para promover la generación, protección y comercialización de los derechos de $\mathrm{PI}$ de las universidades asentadas en la Entidad dedicadas a la I+D+i. Entre los activos de PI que las OTT deben gestionar, destacan: los derechos de autor y los derechos conexos así como la propiedad industrial (patentes, modelos de utilidad, dibujos y modelos industriales, variedades vegetales, marcas y otros signos distintivos e indicaciones geográficas). (Thursby y Kemp, 2002; OMPI, 2006; Siegel, Veugelers y Wright, 2007; Morejón y Velázquez, 20II).

Cabe señalar que las OTT pueden funcionar de acuerdo con su grado de autonomía en tres tipos (Markman et. al. 2005 en Beraza y Rodríguez, 20 10: 166-167):

I. Estructura universitaria tradicional. La cual se integra como departamento o área y su plantilla de personal depende de la IES.

2. Fundación de investigación sin ánimo de lucro. La cual forma parte de una estructura separada de la estructura administrativa universitaria o bien es unidad independiente sin ánimo de lucro, lo cual les permite disponer de su propio presupuesto y su personal forma parte de la plantilla de la Universidad.

3. Entidad privada con ánimo de lucro promovida por la Universidad. Son jurídicamente independientes, por lo que cuentan con su propio director y personal lo que les brinda un mayor grado de autonomía y una mejor orientación comercial.Tienen mayor posibilidad a conseguir financiación así como poder financiar nuevas empresas por lo que se pueden especializar en el desarrollo económico y creación de empresas.

Cabe mencionar que la clasificación anterior no es la única, también existen otras consideraciones para clasificar a las OTT, como pueden ser: la relación institución-investigación, los objetivos esperados de la TT, el número de instituciones a las que se brinda servicio o incluso el nivel de especialización de éstas.

\section{Importancia}

El establecimiento de la OTT reviste importancia por el papel significativo que pueden desempeñar en la gestión eficiente de los activos de propiedad intelectual de las Universidades, así como, en la promoción del conocimiento de éstas (OMPI, 20II).

La transferencia de tecnología puede ser vendida, donada, alquilada, intercambiada, etc., y pueden transferirse tanto las tecnologías blandas como la tecnologías duras , y en algunos casos ambas. La importancia de la TT radica en compartir con las empresas el conocimiento científico y tecnológico generado por las Universidades y por los centros de investigación, esto es, que el conocimiento pueda ser aplicado a otros sectores de la economía y pasar así de la teoría a la práctica.

La TT pretende alcanzar soluciones viables desarrolladas por las Universidades aplicables a problemas específicos de los distintos sectores económicos. Del mismo modo, la TT puede potenciar no sólo el crecimiento y desarrollo económico de las empresas sino del lugar en donde éstas se encuentran establecidas, apoyando a las Universidades en su reconocimiento en el ámbito académico y científico y en la generación de recursos financieros; además de fortalecer el vínculo Universidad-empresa al facilitar la comercialización de los resultados de investigación acercándolos a la población.

Por lo tanto, las Universidades deben participar en el desarrollo de tecnologías esenciales para la rentabilidad y futuro de las empresas. Krishen (2012:195) afirma que la aplicación de tecnologías y metodologías en las empresas y mercados comerciales benefician económicamente a la sociedad. Esas 
tecnologías deben estar destinadas a proporcionar nuevos y mejores productos, procesos de fabricación y servicios para todos los sectores de la economía.

Es esencial, logar un nuevo y avanzado cambio de tecnologías y metodologías que permitan el desarrollo y crecimiento económico del país de manera sostenible y asequible. Tanto el sector privado como el sector público deben darse cuenta del potencial y capacidad de innovación que poseen las Universidades y aprovecharlo a su favor. La TT es clave para potenciar el desarrollo económico que tanto requiere México.

Al mismo tiempo, los investigadores universitarios deben tomar en cuenta nuevas y más amplias formas de aprovechar los recursos -técnicos, financieros, humanos, tecnológicosde las instituciones en las que laboran de tal manera que logren apoyar eficientemente a las empresas que lo requieran. Es preciso identificar el origen de los problemas y sus posibles soluciones para conseguir una efectiva comercialización de tecnologías desarrollas para problemas específicos. De acuerdo con Niels Reimers fundador y director de la Oficina de Licencias de Tecnología (OTL por sus siglas en inglés) de la Universidad de Stanford, los investigadores universitarios deben desarrollar y aplicar tecnologías para el uso y beneficio público para generar ingresos para propósitos de educación y de investigación dentro de las Universidades (Feldman et al., 2007 y Powers y Campbell, 2009).

Debe tomarse en cuenta que con la TT no sólo las empresas se ven beneficiadas sino también las Universidades ya que se allegan de recursos monetarios derivados la comercialización de sus tecnologías. Con dichos recursos las Universidades pueden: aumentar su inversión en $I+D+i-$ financiando nuevos proyectos y nuevas líneas de investigación-, adquirir equipamiento, insumos y material de laboratorio, participar en congresos, comprar membrecías u organizar de eventos Sin embargo, para que la TT universitaria tenga éxito es necesario desarrollar relaciones perdurables con las empresas. Feldman, Colaianni y Liu (2007: 1799) señalan que la firma de un acuerdo de licencia representa una transacción que es un primer paso en una relación que requiere de mantenimiento y supervisión. La vinculación de las Universidades con el sector empresarial facilita la TT, por lo que es necesario intensificar la relación entre éstas, esto es, establecer una relación permanente entre ambos actores. De aquí, que las oficinas de TT faciliten esa tarea. Hidalgo y León (2006) sugieren cuatro condiciones para crear, modificar o transferir conocimiento de las Universidades a otras organizaciones. Estas condiciones son las siguientes:

I. Cualificar alumnos en temas asociados a conocimiento ya consolidado (tanto en las etapas de grado y postgrado universitario).
2. Generar nuevo conocimiento como un producto esencial de su actividad investigadora.

3. Transferir y compartir conocimiento e ideas con las empresas u otras organizaciones públicas y privadas externas (actividad conocida como el tercer papel de la Universidad).

4. Diseminar el conocimiento científico y tecnológico a la sociedad en su conjunto.

5. La recolección comprensiva, la gestión y la diseminación de información tecnológica para apoyar la transferencia de tecnología, la creación de varias bases de datos en línea, portales de tecnología, y la celebración de seminarios $y$ encuentros.

\section{Obstáculos}

Las principales dificultades a las que se enfrenta México se observan a continuación:

- $\quad$ Costos de investigación elevados (OMPI, 20 I I:I I6). - Capacidad financiera restringida en materia de investigación interna

- Desconocimiento de las ventajas de poseer y comercializar los derechos de P.I.

- $\quad$ Ausencia de una política nacional e institucional clara en materia de P.I.

- $\quad$ Falta de incentivos para los investigadores

- Subsidios públicos a la investigación y al desarrollo inexistentes $o$ insuficientes

- Financiación de I+D por otras entidades (donantes, ONG, etc.).

- $\quad$ Precariedad de la infraestructura destinada a la investigación tecnológica

- $\quad$ Falta de políticas contra el éxodo intelectual de especialistas.

- Debilidad del sector industrial nacional.

\section{Beneficios}

Para el gobierno u otras organizaciones públicas o privadas encargadas de financiar proyectos de I+D+i, es necesario conocer la "Vuelta Tecnológica " que reciben de sus contribuciones. Aparte de retorno tecnológico directo, también hay beneficios secundarios para la industria mexicana y para la sociedad en su conjunto. En particular, estos incluyen: beneficios industriales y programas de educación y capacitación. Los beneficios específicos para el sector empresarial son: el desarrollo de nuevos productos, como consecuencia directa del contrato original; la partida de los nuevos equipos de I + D; el inicio de nuevas unidades de negocio; la apertura de nuevos mercados; el aumento de la exposición internacional; el aprendizaje tecnológico; mercado de aprendizaje; aumen- 
to de las ventas; un mayor crecimiento del empleo y mejor rendimiento tecnológico.

Para los programas de educación y capacitación, los beneficios son: becas para la formación de jóvenes investigadores; organización de seminarios, talleres y cursos sobre diversos temas científicos y tecnológicos y la difusión de las investigaciones a través de diversos medios de comunicación.

Entre las ventajas de la adquisición externa de tecnología se hallan el ahorro de tiempo y el ahorro de costos a la empresa, cuando el desarrollo propio de una tecnología de igual calidad requeriría mayores recursos de I+D+i que su adquisición. Acostumbrará a haber ahorro de costos en aquellos casos en que la tecnología disponible en el mercado no requiera demasiadas adaptaciones para su aplicabilidad a la empresa (non context-specific) y cuando los costos de contratación no sean altos (Arbussá, Bikfalvi y Valls, 2004:45).

Panorama de las OTT en México y en el estado de Hidalgo En México existe un creciente interés por desarrollar innovación y hay grandes oportunidades de mercado a nivel global. Sin embargo aún falta cultura tecnológica y mecanismos que encaucen los diferentes esfuerzos al logro de objetivo y con resultados tangibles.

Aunado a lo anterior a pesar de que existe una gran diversidad de organismos en México que dan soporte al desarrollo tecnológico no hay una efectiva interrelación entre ellos por lo que para el desarrollo del tejido empresarial asociado a la transferencia de tecnología se requieren apoyos económicos y una gestión activa de las redes, así como de personal competente para facilitar el proceso lo que redunda en la necesidad de la creación y fortalecimiento de las OTT. Por ello, tomando como base las IES y los Centro de Investigación inscritos en el PNPC del CONACYT, en el siguiente cuadro se enlistan las IES y centros que cuentan con áreas de Vinculación, Unidades de Vinculación o de Transferencia Tecnológica y que han hecho gestiones de propiedad intelectual.

Como se observa en el cuadro anterior, en México aproximadamente un $70 \%$ de las instituciones cuentan con una UVTC incorporada a la estructura universitaria tradicional. Ante este panorama, el gobierno federal de México a través de la Secretaría de Economía y el Consejo Nacional de Ciencia y Tecnología convoca desde el año $201 \mathrm{l}$ a través del "Fondo Sectorial de Innovación Secretaría de EconomíaCONACYT" (FINNOVA) a participar en un programa con la finalidad de fomentar la creación y fortalecimiento de Oficinas de Transferencia de Conocimiento, consultoría, así como un vínculo con inversionistas con la función de identificar o evaluar la viabilidad comercial de los resultados de investigación así como proyectar los resultados esperados de las tecnologías maduradas comercialmente. El FINNOVA además establece que la conformación de las Oficinas de Transferencia de Conocimiento pasarán por tres fases: Pre-Certificación y Certificación con el objetivo de contar con un nivel mínimo de reglamentos y directivas que rijan la transferencia del conocimiento y la fase de apoyos a corto y largo plazo con la finalidad de proveer apoyos económicos a un grupo de beneficiarios certificados para facilitar la maduración de las OTT existentes o en desarrollo.

Algunos Estados de la República Mexicana han comenzado a realizar esfuerzos por incentivar la creación de las OTT, tal es el caso del estado de Nuevo León que buscó consolidar a Monterrey y su Zona Metropolitana como Ciudad Internacional del Conocimiento al formalizar el Instituto de Innovación y Transferencia de Tecnología. En el Estado de Baja California en Diciembre del 20II, el Centro de Investigaciones Biológicas del Noroeste (CIBNOR), el Centro de Investigación Científica y de Educación Superior de Ensenada (CICESE) y Avanza Capital se unieron para constituir la primera UVTC (CICESE, 20I I). Por otra parte en el Estado de Guanajuato se constituirá la primera OTT en el país con socios alemanes y mexicanos quienes en mayo de 20II firmaron convenio. Otro ejemplo es Querétaro donde la Universidad Autónoma de Querétaro en febrero de 2012 informa a su comunidad que un mes después iniciaría operaciones la UVTC en sus instalaciones (UAQ, 20I2).

Sin embargo existen diversos Estados que aún no han alcanzado a visualizar los beneficios e importancia del rol de las OTT, tal es el caso del Estado de Hidalgo cuya participación en el Programa de Estímulos a la Innovación y Desarrollo Tecnológico, que tiene como objetivo motivar a las empresas a que realicen actividades relacionadas con la investigación y desarrollo tecnológico o innovación, tuvo una participación de 15 proyectos por un monto aproximado de 78 millones de pesos, ocupando de esta manera el lugar 13 en el 2010 y en el $201 \mathrm{I}$ participaron 20 proyectos por un monto aproximado de 99 millones de pesos escalando a la posición número II de participación; sin embargo este programa apoya con porcentajes mayores de gastos a las empresas cuando se encuentran vinculadas a una IES o bien a un Centro de Investigación.

Bajo este panorama el estado de Hidalgo ha detectado la necesidad de contar con estructuras que sirvan de puente para facilitar las interacciones entre Universidad-empresagobierno por lo que una primera acción que se ha realizado para impulsar al estado, promover la vinculación y fortalecer los mecanismos institucionales de investigación, ciencia básica y aplicada y tecnología para favorecer la generación, aplicación y desarrollo del conocimiento en áreas estratégicas, fue la modificación al Decreto de Creación del Consejo Estatal de Ciencia y Tecnología del Estado de Hidalgo, pub- 


\begin{tabular}{|c|c|c|c|}
\hline Núm & \begin{tabular}{|l|} 
Entidad \\
Federativa \\
\end{tabular} & Institución & Oficina \\
\hline 1 & Puebla & $\begin{array}{l}\text { Benemérita Universidad Autónoma de } \\
\text { Puebla }\end{array}$ & $\begin{array}{l}\text { Centro Universitario de Vinculación y } \\
\text { Transferencia de Tecnología (CUV) }\end{array}$ \\
\hline 2 & $\begin{array}{l}\text { Distrito } \\
\text { Federal } \\
\end{array}$ & $\begin{array}{l}\text { Centro de Investigación y de Estudios } \\
\text { Avanzados del IPN }\end{array}$ & $\begin{array}{l}\text { Unidad Politécnica para el Desarrollo y } \\
\text { Competitividad Empresarial (UPDCE) }\end{array}$ \\
\hline 3 & Querétaro & Centro Nacional de Metrología & Unidad de Vinculación \\
\hline 4 & $\begin{array}{l}\text { Estado de } \\
\text { México }\end{array}$ & Colegio de Posgraduados & Dirección de Vinculación \\
\hline 5 & Puebla & Fundación Universidad de las Américas & $\begin{array}{l}\text { Dirección General de Tecnologías de la } \\
\text { Información }\end{array}$ \\
\hline 6 & Morelos & Instituto de Investigaciones Eléctricas & $\begin{array}{l}\text { División de Planeación, Gestión de la } \\
\text { Estrategia y Comercialización }\end{array}$ \\
\hline 7 & $\begin{array}{l}\text { Distrito } \\
\text { Federal } \\
\end{array}$ & Instituto Mexicano de Petróleo & $\begin{array}{l}\text { Administración del Conocimiento y Patri- } \\
\text { monio Intelectual (ACPI) }\end{array}$ \\
\hline 8 & Morelos & $\begin{array}{l}\text { Instituto Mexicano de Tecnología del } \\
\text { Agua }\end{array}$ & Información tecnológica y comunicación \\
\hline 9 & Veracruz & Instituto Nacional de Ecología & Departamento de Servicios \\
\hline 10 & $\begin{array}{l}\text { Distrito } \\
\text { Federal }\end{array}$ & $\begin{array}{l}\text { Instituto Nacional de Investigaciones } \\
\text { Forestales, Agrícolas y Pecuarias }\end{array}$ & $\begin{array}{l}\text { Grupo Ganadero para la Validación y } \\
\text { Transferencia de Tecnología (GGVATT) }\end{array}$ \\
\hline 11 & $\begin{array}{l}\text { Distrito } \\
\text { Federal } \\
\end{array}$ & $\begin{array}{l}\text { Instituto Nacional de Investigaciones } \\
\text { Nucleares }\end{array}$ & Dirección de Servicios Tecnológicos \\
\hline 12 & $\begin{array}{l}\text { Distrito } \\
\text { Federal } \\
\end{array}$ & Instituto Politécnico Nacional & $\begin{array}{l}\text { Unidad Politécnica para el Desarrollo y } \\
\text { Competitividad Empresarial (UPDCE) }\end{array}$ \\
\hline 13 & Nuevo León & $\begin{array}{l}\text { Instituto Tecnológico y de Estudios Supe- } \\
\text { rior de Monterrey }\end{array}$ & Centro de Propiedad Intelectual \\
\hline 14 & Coahuila & $\begin{array}{l}\text { Universidad Autónoma Agraria Antonio } \\
\text { Narro }\end{array}$ & $\begin{array}{l}\text { Subdirección de Difusión Científica y } \\
\text { Tecnológica }\end{array}$ \\
\hline 15 & $\begin{array}{l}\text { Baja Califor- } \\
\text { nia }\end{array}$ & Universidad Autónoma de Baja California & $\begin{array}{l}\text { Centro de Desarrollo y Transferencia de } \\
\text { Tecnología (CDTT) }\end{array}$ \\
\hline 16 & Nayarit & Universidad Autónoma de Chapingo & $\begin{array}{l}\text { Centro de Validación y Transferencia de } \\
\text { Tecnología Agropecuaria }\end{array}$ \\
\hline 17 & Chiapas & Universidad Autónoma de Chiapas & $\begin{array}{l}\text { Unidad Incubadora de Negocios INCU- } \\
\text { BO }\end{array}$ \\
\hline 19 & Nuevo León & Universidad Autónoma de Nuevo León & $\begin{array}{l}\text { Centro de Innovación, Investigación y } \\
\text { Desarrollo en Ingeniería y Tecnología }\end{array}$ \\
\hline 20 & \begin{tabular}{|l|} 
San Luis \\
Potosí
\end{tabular} & $\begin{array}{l}\text { Universidad Autónoma de San Luis } \\
\text { Potosí }\end{array}$ & $\begin{array}{l}\text { Centro Universitario y de Apoyo Tec- } \\
\text { nológico y Empresarial (CUATE) }\end{array}$ \\
\hline 21 & Tamaulipas & Universidad Autónoma de Tamaulipas & Secretaría de Extensión y Vinculación \\
\hline 23 & $\begin{array}{l}\text { Estado de } \\
\text { México } \\
\end{array}$ & $\begin{array}{l}\text { Universidad Autónoma del Estado de } \\
\text { México }\end{array}$ & $\begin{array}{l}\text { Departamento de Promoción y Desarrollo } \\
\text { de la Vinculación }\end{array}$ \\
\hline 24 & Morelos & $\begin{array}{l}\text { Universidad Autónoma del Estado de } \\
\text { Morelos }\end{array}$ & $\begin{array}{l}\text { Unidad de Gestión y Transferencia de } \\
\text { Tecnología }\end{array}$ \\
\hline 25 & Colima & Universidad de Colima & Coordinación General de Vinculación \\
\hline 26 & Jalisco & Universidad de Guadalajara & $\begin{array}{l}\text { Coordinación de Vinculación y Servicio } \\
\text { Social }\end{array}$ \\
\hline
\end{tabular}




\begin{tabular}{|c|c|c|c|}
\hline 27 & Guanajuato & Universidad de Guanajuato & $\begin{array}{l}\text { Centro Universitario Vinculación con el } \\
\text { Entorno }\end{array}$ \\
\hline 28 & Oaxaca & Universidad del Mar & $\begin{array}{l}\text { Coordinación de Promoción del desar- } \\
\text { rollo }\end{array}$ \\
\hline 30 & Tabasco & Universidad Juárez Autónoma de Tabasco & $\begin{array}{l}\text { Coordinación de Vinculación y Educación } \\
\text { Continua }\end{array}$ \\
\hline 32 & $\begin{array}{l}\text { Distrito } \\
\text { Federal } \\
\end{array}$ & $\begin{array}{l}\text { Universidad Nacional Autónoma de } \\
\text { México }\end{array}$ & $\begin{array}{l}\text { Unidad de Vinculación y Transferencia de } \\
\text { Tecnología }\end{array}$ \\
\hline 33 & Puebla & $\begin{array}{l}\text { Universidad Popular Autónoma del Esta- } \\
\text { do de Puebla }\end{array}$ & Dirección de Investigación \\
\hline 34 & Oaxaca & Universidad Tecnológica de la Mixteca & $\begin{array}{l}\text { Coordinación de Promoción del Desar- } \\
\text { rollo }\end{array}$ \\
\hline 35 & Hidalgo & Universidad Tecnológica de Tula Tepeji & $\begin{array}{l}\text { Centro Incubador de Empresas de la Uni- } \\
\text { versidad Tecnológica de Tula-Tepeji }\end{array}$ \\
\hline 36 & Veracruz & Universidad Veracruzana & Dirección de Vinculación General \\
\hline 37 & Hidalgo & $\begin{array}{l}\text { Universidad Autónoma del Estado de } \\
\text { Hidalgo }\end{array}$ & $\begin{array}{l}\text { División de Vinculación (área de Trans- } \\
\text { ferencia de Tecnología) }\end{array}$ \\
\hline
\end{tabular}

Cuadro I. Instituciones de Educación Superior y Centros de Investigación con Oficina de Vinculación o de Transferencia Tecnológica. Fuente: elaboración propia con bases en UNAM, 2012.

licado en el Diario Oficial de la Federación con fecha del 20 de mayo de 2002 donde en su artículo 5 fracción X, se establece que el Consejo de Ciencia y Tecnología del Estado de Hidalgo tendrá la facultad de "celebrar acuerdos y convenios de colaboración con organizaciones privadas y sociales, para el desarrollo de proyectos de carácter científico y desarrollo o transferencia tecnológicos e innovación" y en su fracción XV de "realizar, promover y difundir estudios, investigaciones y transferencia de tecnología, para formar un sistema de información, registro, seguimiento y evaluación de la investigación científica, el desarrollo tecnológico, incluyendo en dicho sistema al posgrado". (COCYTEH, 20I3). Lo anterior fundamenta la creación de la Oficina de Transferencia de Conocimiento, la cual tiene como misión "Colaborar con instituciones de investigación y desarrollo tecnológico para detectar las necesidades y oportunidades de transferencia de conocimiento y tecnología, vinculándolas al desarrollo económico del estado de Hidalgo, impulsando la correcta toma de decisiones de clientes y asociados, e impactando la cadena de valor desde el requerimiento tecnológico hasta la fuente de investigación y desarrollo. Promoviendo el apoyo y orientación profesional en las áreas de formación empresarial, servicios de transferencia de tecnología y nuevas empresas de base tecnológica; a investigadores para la comercialización y explotación de los resultados de su investigación, actuando como vínculo entre las entidades externas y otras instituciones académicas, con inversionistas y compañías para la transferencia de tecnología" (COCYTEH, 2013). En principio, ha establecido convenio de colaboración con II Instituciones de Educación Superior del Estado y ofrece una cartera de servicios a instituciones destacando la asesoría y consultoría para la TT, gestoría para la obtención de fondos, asesoría para el desarrollo de proyectos de $\mathrm{I}+\mathrm{D}+\mathrm{i}$, de programas de marketing interno $\mathrm{y}$ para el desarrollo de OTC institucionales, entre otros; contando con un respaldo de políticas en materia de $\mathrm{Pl}$, consultoría, de rechazo de licenciamiento, tiempo de respuesta y conflictos de interés.

Por lo que se muestra un claro avance de la importancia del papel de la OTT en el Estado de Hidalgo como mediador en la relación de la triple hélice por una convivencia socialmente responsable entre la ciencia y la industria, por ello las entidades y sus principales Universidades deberán definir la misión de las OTT, la transparencia en sus políticas y procedimientos y su esquema de participación pues de ello dependerá su modelo de operación, entre otros factores que impactan en el éxito.

En resumen es necesario construir puentes de enlace que sirvan para establecer el andamiaje de un ecosistema de innovación estatal y nacional eficiente.

\section{Conclusiones}

La necesidad de crear nuevas tecnologías e innovar en las ya existentes es una constante en la vida diaria, además, de ser la fuerza impulsora detrás del desarrollo tecnológico y de la transferencia tecnológica para cualquier país. 
La TT se encuentra asociada con diversos aspectos, tales como: el desarrollo económico, la competitividad de las empresas y el éxito de las organizaciones. Por tal razón, fomentar la creación OTT's dentro de las Universidades involucra un esfuerzo en conjunto entre éstas, las empresas y el gobierno ya que la $I+D+i$ implica elevados costos que pueden ser abatidos con las alianzas entre los actores antes mencionados. Sin embargo, no todas las Universidades cuentan con una OTT que se encargue de gestionar adecuadamente el conocimiento que desarrollan sus investigadores y que pueda ayudarlas a solucionar los múltiples problemas $\mathrm{u}$ obstáculos a los que se enfrentan los proyectos tecnológicos. Lo anterior puede ser perjudicial para la institución por los siguientes motivos: los proyectos de $\mathrm{I}+\mathrm{D}+\mathrm{i}$ podrían no ser rentables y no contar con un mercado meta; los investigadores trabajan con el desconocimiento de las necesidades de los empresarios y/o de la sociedad; los proyectos de I+D+i podrían no ser viables; no contar con el suficiente financiamiento para desarrollar nuevas líneas de investigación; no contar con los recursos suficientes para comercializar sus tecnologías.

Como puede observarse las OTT poseen un papel importante para lograr contrarrestar los aspectos antes mencionados y generar beneficios tanto para las empresas, las Universidades y por supuesto para la sociedad. Tal y como se señala en esta investigación existen obstáculos y riesgos para la TT, mismos que pueden ser abatidos por la gestión eficiente de las OTT.

Se espera que las OTT sean el motor de la economía, ya que han atraído la atención de instituciones de renombre como el CONACYT o la OMPI, así como de comunidades académicas locales para crear mecanismo que ayuden a eficientar su gestión.

Las OTT deben permanecer en la constante búsqueda para desarrollar prácticas más eficientes que permitan a las Universidades adaptarse al dinámico entorno en el que se encuentran y satisfacer así tanto las expectativas internas -investigadores, autoridades- como las externas -empresarios y sociedad-. El ámbito académico y el empresarial deben desarrollarse en conjunto para intentar satisfacer las necesidades sociales y lograr un entorno económico, sociocultural y natural favorable para el país.

\section{Bibliografía}

ACOSTA, M., y Coronado, D. (1999). Innovación tecnológica y desarrollo regional. Información Comercial Española, ICE: Revista de Economía, (78I), 103-II6.

ARBUSSÁ,A., Bikfalvi,A.y Valls, J. (2004). La I+D en las pymes: Intensidad y estrategia. Universia business review-actualidad económica.

Asociación Mexicana de Directivos de la Investigación Aplicada y el Desarrollo Tecnológico, A.C., ADIAT (20I0). Oficinas de Transferencia de Tecnología. Fundamentos para su formación y operación en México. México. P. 34.

AVARO, D. (2006). Cátedra ALCUE. Sociedad del conocimiento. FLACSO, México.

BERAZA, G. y Rodríguez, A. (2010). Estructuras de Intermediación para la Transferencia de Conocimiento Universitario: Las Oficinas de Transferencia Tecnológica. Propiedad Intelectual, núm. I3. Universidad de los Andes, Venezuela. Pp. I52-176.

BUENO, E. (2007). La tercera misión de la Universidad: El reto de la Transferencia del conocimiento. Revista madrid+d, No. 41 .

Centro de Investigación Científica y de Educación Superior de Ensenada (CICESE), 201I. "Se crea la primera Unidad de Vinculación y Transferencia del Conocimiento". http://todos.cicese.mx/index. php?option $=$ com_content $\&$ view $=$ article $\& i d=276 \% 3$ Asecrea-la-primera-unidad-de-vinculacion-y-transferencia-delconocimiento\&catid $=9 \% 3$ Abreviario\&ltemid $=77$ (Consultado julio 27, 20I2).

CIMOLI, M. (2000). Developing Innovation Systems: Mexico in a Global Context. New York, USA.

CIMOLI, M. y Dosi, G. (1995), “Technological paradigms, patterns of learning and development: an introductory roadmap". Journal of Evolutionary Economics. Núm.5, pp. 243268.

CLOSS, L., Ferreira, G., Soria, A., Sampaio, C., \& Perin, M. (20I2). Organizational Factors that Affect the University-Industry Technology Transfer Processes of a Private University. Journal Of Technology Management \& Innovation, 7(I).

Comité Intersectorial para la Innovación, Cll (20II). Programa Nacional de Innovación. http://www.adiat.org/es/documento/402.pdf (Consultado el 19 de agosto, 20I2).

ISSN: 07 I8-2724. (http://www.jotmi.org) 
Consejo de Ciencia y Tecnología del Estado de Hidalgo. Decreto de Creación del COCYTEH. http://siicyteh.hidalgo. gob.mx/oficina/oficina.html (Consultado el 02 de mayo, 2013).

Consejo de Ciencia y Tecnología del Estado de Hidalgo. Misión, servicios, alianzas con instituciones de la Oficina de Transferencia de Conocimiento. http://siicyteh.hidalgo.gob. $\mathrm{mx} /$ oficina/oficina.html (Consultado el 02 de mayo, 20I3).

Consejo Nacional de Ciencia y Tecnología, CONACYT (20I0). Glosario de términos Subprograma AVANCE. Diario Oficial de la Federación (2002). Ley de Ciencia y Tecnología. http://www.diputados.gob.mx/LeyesBiblio/ref/lct.htm (Consultado agosto 18,2012 ).

Consejo Nacional de Ciencia y Tecnología, CONACYT (20I2a). Programa Especial de Ciencia, Tecnología e Innovación (PECiTI). http://www.siicyt.gob.mx/siicyt/docs/contenido/PECiTI.pdf (Consultado septiembre I3, 20I2).

Consejo Nacional de Ciencia y Tecnología, CONACYT (20/2b). Programas de apoyo a la innovación. http://www. pm7ue.conacyt.cinvestav.mx/Portals/0/SiteDocs3P/Presentaciones/MI/Cooperacion/5_Apoyolnnovacion.pdf (Consultado septiembre 17,20I2)

Consejo Nacional de Ciencia y Tecnología, CONACYT (20I3). Oficinas de Transferencia de Tecnología. Fondos y apoyos CONACYT.

DE SÁ NUNES,A.;Augusto,A. y Segatto,A. (2009). Papéis de un escritório de Transferência de Tecnologia: Comparação entre Universidades privada e pública. Simpoi de Administração da Produção, Logística e Operações Internacionais.

FAGERBERG, J. Srholec, M., y Verspagen, B. (2010). Innovation and economic dvelopment. Handbook of the Economics of Innovation, 2, 833-872.

FELDMAN, M., Colaianni, A. \& Liu, C. (2007) Lessons from the Commercialization of the Cohen-Boyer Patents: The Stanford University Licensing Program.

Foro Consultivo Científico y Tecnológico, FCCyT (2012). Diagnóstico en Ciencia, Tecnología e Innovación 2004-20I0. Hidalgo. http://www.foroconsultivo.org.mx/libros_editados/diagnosticos2/hidalgo.pdf (Consultado septiembre 20, 20I2).

Foro Consultivo Científico y Tecnológico, FCCyT (2013). Propuestas para contribuir al Diseño del PECiTI 2012-2037, documento de trabajo del FCCyT, AC, México. http://www. foroconsultivo.org.mx/documentos/peciti_2012_2037/4_ dinamica_de_innovacion.pdf (Consultado Mayo 09, 2013).
FREEMAN, C. (1987). Technology policy and economic performance: Lessons from Japan. London: Pinter.

FREEMAN, C. (I99|). Networks of Innovation:A review and Introduction to the Issues. Research Policy, 20 (5), Pp. 499514.

Fundación Cotec para la Innovación Tecnológica (2003). Nuevos mecanismos de transferencia de tecnología debilidades y oportunidades del Sistema Español de Transferencia de Tecnología. Madrid.

GACEL Ávila, J. (2000). La dimensión internacional de las Universidades mexicanas. Revista de la Educación Superior. Publicación de la Asociación Nacional de Universidad e Instituciones de Educación Superior. http://www.anuies.mx/ servicios/P_anuies/publicaciones/revsup/res I I5/art6.htm (Consultado agosto 6, 20I2).

Gobierno de los Estados Unidos Mexicanos, Presidencia de la República. (2007). Plan Nacional de Desarrollo, PND. http://www.snieg.mx/contenidos/espanol/normatividad/ marcojuridico/PND_2007-20I2.pdf Consultado agosto 19, 2012)

Grupo Interuniversitario de Investigación y Análisis Estratégico para el Desarrollo de la Pyme (2008). "Reunión técnica internacional creación red de investigación en pymes". Universidad de Cantabria, España.

GURMU, S., Black, G. y Stephan, P. (2010). The knowledge production function for university patenting. Economic Inquiry, Vol. 48, No. I, January 2010, pp. I92-2I3.

HAMDAN, H., Yusof, F. Omar, D., Abdullah, F. Nasrudin, N. y Abullah, I. C. (20I I). University Industrial Linkages: Relationship Towards Economic Growth and Development in Malaysia. World Academy of Science, Engineering and Technology No. 58.

HIDALGO, A. y León, G. (2006). La importancia del conocimiento científico y tecnológico en el proceso innovador.

HÜLSBECK, M., Lehmann, E. y Starnecker, A. (20II) Performance of technology transfer offices in Germany. The Journal of Technology Transfer.Vol. 38, Issue 3, June, 20 I3. pp. 199-215

Índice de Innovación Estatal, I2E (2010). http://www.arinformacion.com/mexico/docs/publicaciones/IIE_2010.pdf (Consultado agosto 30,2012 )

Instituto Mexicano para la Competitividad, IMCO (2008). Ranking por Estados. http://imco.org.mx/es/ranking/estados/ (Consultado agosto 30, 20I2). 
Instituto Nacional de Estadística y Geografía, INEGI (2009). Censo Económico 2009. http://www.inegi.org.mx/est/contenidos/espanol/proyectos/censos/ce2009/pdf/RD09-resumen.pdf (Consultado agosto I3, 2012).

KAUFFMAN, S. (200I). El desarrollo de las micro, pequeñas y medianas empresas: un reto para la economía mexicana. Universidad Veracruzana. http://www.uv.mx/iiesca/revista/ documents/empresas200I-I.pdf (Consultado julio 23, 20I2)

KRISHEN, K. (20I2). Multiple Aspects of Space Technology Transfer. Advanced Planning Office, NASA Johnson Space Center.

KRÜCKEN, G., Kosmützky, A. y Torka, M. (2007). Towards a multiversity? Universities between global trends and national traditions (Pp. I08-13I) Bielefeld:Transcript.

METCALF, S. (1995). The Economic Foundations of Technology Policy' in P. Handbook of the Economics of Innovation and Technical Change. Oxford: Blackwell.

MOREjÓN, M. y Velázquez, R. (20ll). Propuesta de una tecnología para la gestión de la propiedad intelectual en la empresa estatal cubana. Revista Cubana de ACIMED.Vol. 22, núm. 3, pp. 204-218.

MOWERY, D., Nelson, R., Sampat, B. and Ziedonis, A. (2004). Ivory Tower and Industrial Innovation: University- Industry Technology Transfer Before and After the Bayh-Dole Act. Stanford, CA: Stanford Business Books, an imprint of Stanford University Press.

NELSON, R. (ed.) (1993), "National systems of innovation", Nueva York, Oxford University Press.

NELSON, R. y Winter, S. (1982). An Evolutionary Theory of Economic Change. Cambridge, MA: Harvard University Press.

NEPAL, C., Raj, B. y Prasad, K. (2004). Technology transfer in SMEs: problems and issues in the context of Nepal. Trade and Investment Division (TID), Bangkok, Thailand. http:// www.unescap.org/tid/publication/indpub2306.pdf (Consultado julio I2, 20I2).

Observatorio Virtual de Transferencia de Tecnología, OVTT (2012). Oficinas de Transferencia de Tecnología y Parques Científicos. http://www.ovtt.org/mercado/oficinas (Consultado agosto 7,2012 )

Organización Mundial de la Propiedad Intelectual, OMPI (2006). La propiedad intelectual en la Universidad: aplicación de las políticas. Revista de la OMPI. Núm. 5, Ginebra.
Organización Mundial de Propiedad Intelectual, OMPI (20I I). Guía práctica para la creación y la gestión de oficinas de transferencia de tecnología en Universidades y centros de investigación de América Latina. Powers, J. y Campbell, E. (2009) University Technology Transfer in Tough Economic Times.

Organización Mundial de Propiedad Intelectual, OMPI (20I2). WIPO IP Facts and Figures. http://www.wipo.int/ freepublications/en/statistics/943/wipo_pub_943_20I2.pdf (Consultado julio 7, 20I2).

Registro Nacional de Instituciones y Empresas Científicas y Tecnológicas, RENIECYT (20I2). http://www.conacyt.gob. $\mathrm{mx} /$ registros/reniecyt/Paginas/Reniecyt_Estadisticas.aspx (Consultado mayo 5, 20I2).

RODRÍGUEZ, D. (2005). El proceso de Transferencia de Tecnología. Documento de Trabajo, Universidad Nacional Autónoma del México. Pp. 4-5

SALAZAR, E., Romero, C. y Carranza, Y. (2010). Tecnología y formación. Una reflexión desde la facultad de tecnología.

SÁNCHEZ, G. (2007). Perspectivas de las micro y pequeñas empresas como factores del desarrollo económico de México. http:// I32.248.45.5/profesor/barajas/perspec.pdf (Consultado julio 20, 20I2).

SANCHO, R. (2007). Innovación industrial. Revista Española De Documentación Científica.

SIEGEL, D., Veugelers, R. y Wright, M. (2007). Technology transfer offices and commercialization of university intellectual property: performance and policy implications. Oxford Review of Economic Policy.Vol. 23, Núm. 4. Pp. 640-660.

SOLÉ, F.,Valls, J., Condom, P., Bikfalvi,A., Pérez,A.y X.Amores (2003). Ėxit de Mercat i Innovació. Departamento de Trabajo, Industria, Comercio y Turismo de la Generalitat de Cataluña. Centro para la Innovación y el Desarrollo Empresarial (ClDEM). Barcelona.

TAPIA, A. y Capdeville, M. (1998). Corrientes y conceptos de la teoría evolucionista, en Corona, L. Teorías económicas de la tecnología. IPN, México.

TECUANHUEY, E. (2007). La innovación en tecnologías blandas: condición de la innovación tecnológica en el sector servicios. http://www.uasnet.mx/ridit/Congreso2007/ m3p07.pdf (Consultado julio 2, 20I2).

THURSBY, J. y Kemp, S. (2002). Growth and productive efficiency of university intellectual property licensing. Research Policy.Vol.3I, Núm. I, pp. 109-124. 
Universidad de Medellín, 2012. Investigación http://www. udem.edu.co/UDEM/Investigacion/CentrosDelnvestigacion/ (Consultado junio 30, 20I2).

Universidad Nacional Autónoma de México. Dirección General de Evaluación Institucional. (2012) http://www.dgei. unam.mx/?q=node/6 I (Consultado agosto 20, 20I2). 